\title{
Simulation and Fabrication of Broadband Tunable Phase Shifter Based on Transmission Line Metamaterial
}

\author{
Su SHENG, Hanxiao CHEN, Jian WEN, Hongri LIU \\ College of Physics and Electronic Science, Hubei Normal University, Huangshi 435002, China \\ whsheng2004@126.com
}

Submitted July 2, 2018 / Accepted June 2, 2019

\begin{abstract}
This paper presents a kind of broadband, lowloss tunable phase shifter based on transmission line metamaterial. An inherent high-pass backward-wave response property of a left-handed metamaterial based on coplanar waveguide $(C P W)$ transmission line was used to realize a microwave phase shifter. The commercial software Ansoft HFSS was used to design and analyze for the transmission line metamaterial phase shifter structure, and the resulting S-parameters were used to characterize its performance. A transmission line metamaterial phase shifter was fabricated on Copper-Clad Board. The designed four unit-cells phase shifter provides a $0-190^{\circ}$ continuous phase shift at $7.2 \mathrm{GHz}$ using varactors biased from $0 \mathrm{~V}$ to $6 \mathrm{~V}$ with relatively low insertion loss.
\end{abstract}

\section{Keywords}

Transmission line metamaterial, tunable phase shifter, varactor, broadband

\section{Introduction}

Metamaterials are artificial structures that are designed to exhibit specific electromagnetic properties required for different applications but not commonly found in nature. Recently, the idea of complex materials in which both the permittivity and the permeability possess negative real values at certain frequencies has received considerable attention. In 1968, Veselago theoretically investigated plane-wave propagation in a material whose permittivity and permeability were assumed to be simultaneously negative [1]. In order to implement metamaterials with negative permittivity and permeability, a number of structures have been presented, such as the array of split ring resonators and thin metal wire, $\Omega$-like or S-like ring array, transmission lines with series capacitors and shunt inductors loading, as well as those with split ring resonators or complementary split ring resonators loading [2-10]. Transmission line metamaterials using the possibility of forward and backward wave propagation or the independent design of the phase and impedance [11-13], have a broad range of potential applications. Such artificial transmission lines can be designed with a large positive or negative phase shift making it possible to realize compact filters, phase shifters, and couplers [14-16]. Leaky-wave antennas with a frequency tunable radiation covering $180^{\circ}$ including the broadside direction in its fundamental spatial harmonic have been demonstrated [17]. By employing transmission line metamaterials with lumped elements, it is possible to tailor the electric and magnetic response independently of each other for a desired application. Furthermore, with tunable elements, components with reconfigurable properties can be realized. It was found in the transmission line metamaterials, varactor diodes can give rise to the tunability as they are very sensitive to a low direct current voltage, for instance, leaky-wave antenna with tunable radiation angle and beamwidth, tunable directional coupler, and so on [18], [19]. Specially, transmission line metamaterials with varactor diodes can even serve as model systems for investigating several nonlinear phenomena in negativeindex metamaterials, like asymmetric parametric amplification, abnormal pulse formation, dark Schrodinger solitons and harmonic generation [20], [21]. Traditionally, the lefthanded transmission line metamaterials have been fabricated by loading a host transmission line with series capacitance and shunt inductance. The unit cell of such a loaded transmission line is shown in Fig. 1. Some prominent electronic implementations of left-handed transmission line metamaterial phase shifters include microfluidic channels [22], mechanically variable MIM capacitors [23], CMOS-based active inductors [24], BST thick-film substrates [25], GaAs [26] and Schottky varactor diodes [27], ferroelectric varactors [28], and microelectromechanical systems (MEMS) capacitors [29]. Table 1 summarizes the performance comparison of various transmission line metamaterial phase shifters reported in the literature to date [30]. The capacitors can be replaced by varactors so that with a simple biasing network a tunable transmission line can be implemented and the phase and impedance can be tuned for the application in transmission line components. These transmission line metamaterials exhibit an inherent high-pass backward-wave response in their lowest harmonics, leading to novel microwave devices that are compact and wide-band in nature. However, the practical use of such transmission line metamaterials still poses a number of technological and theoretical challenges in terms of ope- 


\begin{tabular}{|c|c|c|c|c|c|c|}
\hline Technology & Microfluids [22] & $\begin{array}{c}\text { Metallic } \\
\text { perturber [23] }\end{array}$ & $\begin{array}{c}\text { MMIC } \\
\text { inductor \& } \\
\text { varactor diode } \\
{[24]}\end{array}$ & $\begin{array}{c}\text { Varactor } \\
\text { diode [28] }\end{array}$ & PIN diode [30] & This work \\
\hline Size $(\mathrm{mm} \times \mathrm{mm})$ & $30.0 \times 68.0$ & $85.5 \times 30.0$ & $10.8 \times 9.4$ & $30.0 \times 40.0$ & $17.8 \times 21.6$ & $20.0 \times 32.0$ \\
\hline Frequency $(\mathrm{GHz})$ & 0.9 & 2.0 & 2.5 & 17 & 1.0 & 7.2 \\
\hline Insertion loss $(\mathrm{dB})$ & $4.0-5.6$ & $0.56-0.69$ & $0.55-1.1$ & $4.0-5.0$ & $0.89-1.43$ & $1.3-2.8$ \\
\hline Phase range $\left(^{\circ}\right)$ & $(-22)-30$ & $313-355$ & $(-40)-34$ & $51-58$ & $(-14)-59$ & $0-190$ \\
\hline Bandwidth $(\mathrm{GHz})$ & $0.83-0.95$ & $2.00-2.08$ & $2.40-2.70$ & $15.0-20.0$ & $0.92-1.11$ & $7.20-8.70$ \\
\hline
\end{tabular}

Tab 1. Performance comparison of various transmission line metamaterial phase shifters.

rational bandwidth, losses, realization complexity, tuning speed, and tolerance sensitivity.

In this paper, microwave phase shifter based on varactor loaded transmission line metamaterial is presented. The commercial software Ansoft HFSS was used to design and analyze a transmission line metamaterial phase shifter structure. Commercial available lumped elements varactor and inductor were used to construct a unit cell of transmission line metamaterial. The transmission line metamaterial phase shifter exhibited good linear dependence of the differential phase shift with the bias voltage.

\section{Design and Simulation of the Proposed Metamaterial Phase Shifter}

The conventional right-handed $(\mathrm{RH})$ and left-handed (LH) transmission line structures have been theoretically analyzed in the literature and their dispersion properties are well known [31], [32]. Relation between equivalent circuit elements and transmission line characteristic impedance and electrical length at the central frequency are determined by equations derived by comparing ABCD-matrices of transmission line and corresponding distributed T-network structure.

Figure 1 shows the equivalent circuit representation for an infinitesimal short ideal lossless LH transmission line. In this structure, the series capacitor is split up equally into two elements for symmetry reasons. The propagation constant $\beta_{\mathrm{LH}}$ of the LH transmission line unit-cell according to Fig. 1 (neglecting the parasitic RH contributions) are given as

$$
\sin ^{2} \beta_{\mathrm{LH}}=\frac{2}{\omega^{2} L^{\prime} C^{\prime}}
$$

with the inductance and capacitance times unit length defined as

$$
L^{\prime}=L \cdot l, C^{\prime}=C \cdot l
$$

Figure 2 shows the equivalent circuit of a tunable phase shifter, where the series capacitor of LH transmission line unit-cell is split up equally into two elements for

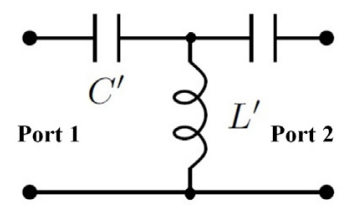

Fig. 1. Equivalent circuit representation for an infinitesimal short ideal lossless LH transmission line.

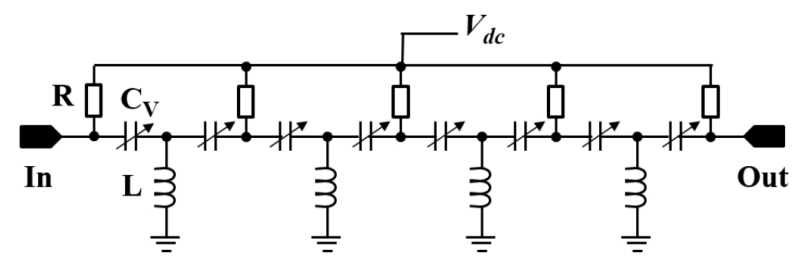

Fig. 2. Equivalent circuit of the tunable phase shifter with four transmission line unit-cells.

symmetry reasons. If several unit cells are cascaded together, there is a cutoff frequency (also known as the Bragg frequency, $\omega_{\mathrm{B}}$ ) near the point where the guided wavelength approaches the periodic spacing of the discrete components [33]

$$
\omega_{\mathrm{B}}=\frac{1}{\sqrt{2 L C}}
$$

where $L$ and $C$ are the inductance and capacitance of one unit-cell. Because this is a high-pass filter structure, the operation frequency must be higher than the Bragg frequency of the transmission line. Above the Bragg frequency, the propagation takes place as a backward wave or in the LH mode because the series and shunt branches are capacitive and inductive respectively. This is the fundamental band of the periodic structure. Then equation (1) can be simplified as

$$
\beta_{\mathrm{LH}}=-\frac{\sqrt{2}}{\omega l \sqrt{L C}}
$$

which leads to a phase shift of

$$
\varphi_{\mathrm{LH}}=\beta_{\mathrm{LH}} \cdot l=-\frac{\sqrt{2}}{\omega} \sqrt{\frac{1}{L C}} .
$$

The easiest way to obtain a tunable device is to replace the capacitor by a varactor $\left(C_{\mathrm{V}}\right)$. That's why when 
talking about tuning from here on it is always meant to tune a capacitor. The unit-cell shows larger absolute values for the phase constant as well as for the tunability

$$
\Delta \varphi_{\mathrm{LH}}=\varphi_{2}-\varphi_{1}=-\frac{\sqrt{2}}{\omega \sqrt{L}}\left(\frac{1}{\sqrt{C_{\mathrm{V} 2}}}-\frac{1}{\sqrt{C_{\mathrm{V} 1}}}\right)
$$

in certain frequency region, where $\varphi_{1}$ and $\varphi_{2}$ denote the insertion phase for two different tuning states. As shown in Fig. 2, the inductors are grounded while the capacitors are placed in series to make up the full LH circuit. On top of the LH circuit there are some resistors that are placed there for application of a voltage bias. This enables users to tune the phase change of the phase shifter. The resistors enable a voltage change from the point of applied voltage to the top of the LH circuit.

In order to predict the response of the metamaterial phase shifter structure, Ansoft's HFSS (Finite Element Method), a commercial 3D modeling package, was used. The entire phase shifter is constructed on defined PCB material with $\varepsilon_{\mathrm{r}}=4.7$ and $h=1.66 \mathrm{~mm}$. The metamaterial phase shifter structure was modeled in the HFSS environment. The completed model of a metamaterial transmission line phase shifter with 4 unit cells is shown in Fig. 3. Finite conductivity (copper) was applied for the ground-signal (GS)

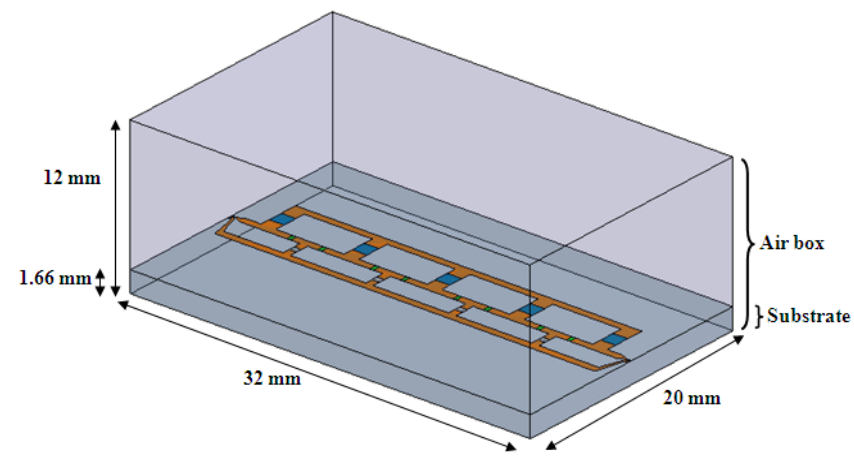

Fig. 3. Model setup of the metamaterial phase shifter in HFSS with detailed dimensions.

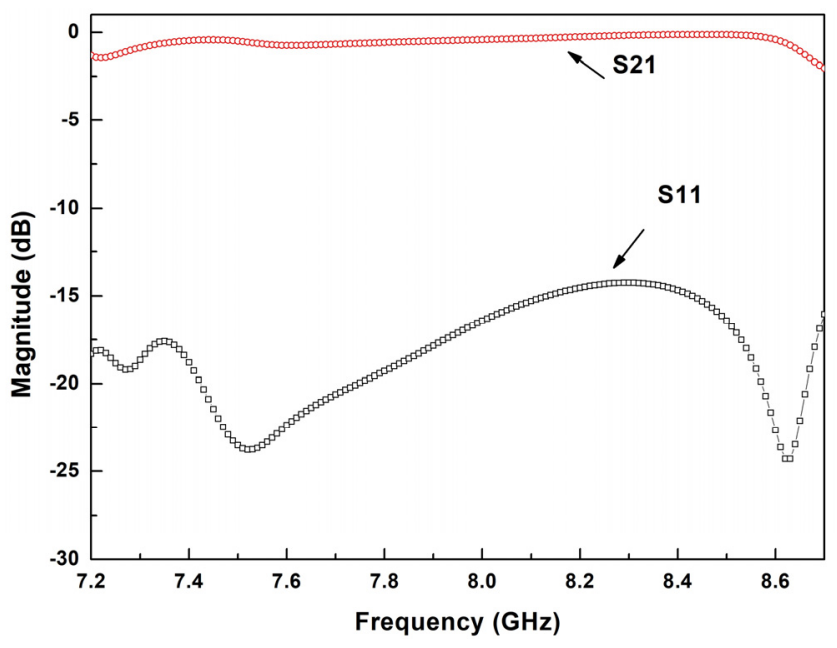

Fig. 4. Simulated reflection coefficient and insertion loss of the metamaterial phase shifter.

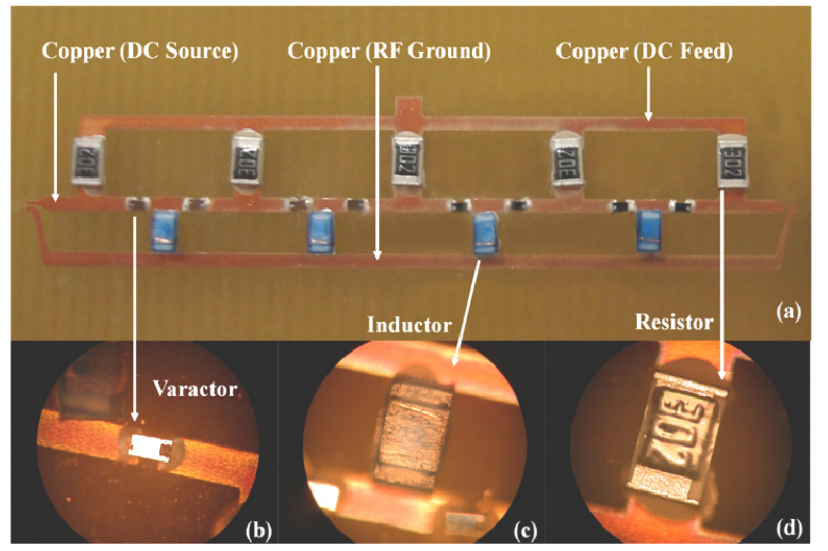

Fig. 5. The magnified photographs of (a) the fabricated transmission line metamaterial phase shifter, (b) a varactor, (c) an inductor, and (d) a resistor.

coplanar waveguide of the phase shifter structure. The lumped RLC boundaries were set to $3 \mathrm{k} \Omega, 1.25 \mathrm{nH}$, and $1.1 \mathrm{pF}$, respectively. The Air box in the HFSS environment was set up with the dimensions of length $=32 \mathrm{~mm}$, width $=$ $20 \mathrm{~mm}$, and height $=12 \mathrm{~mm}$. Figure 4 shows excellent magnitude responses of S11 and S21. In the frequency range of $7.2 \mathrm{GHz}$ to $8.7 \mathrm{GHz}, \mathrm{S} 11$ is less than $-15 \mathrm{~dB}$ and S21 is more than $-2 \mathrm{~dB}$, which means the proposed transmission line metamaterial phase shifter structure has a small insertion loss and offers a great wide bandwidth advantage.

\section{Fabrication and Measurement}

The layout of phase shifter with 4 unit cells based on varactor loaded transmission line metamaterial is shown in Fig. 3. The entire circuit has been implemented using coplanar waveguide geometry. In a normal microwave circuit, the characteristic impedance $Z_{0}$ is set to $50 \Omega$. This is to prevent any impedance mismatch between the microwave source and the circuit that will result in wave reflections and inefficient energy transfer. Based on the simulation results, the inductor and varactor values are approximately $1.25 \mathrm{nH}$ and $1.1 \mathrm{pF}$, respectively. As mentioned above, the resistors are present only for the purpose of application of bias. The metamaterial transmission line phase shifter was realized on a piece of Copper-Clad Circuit Board PCB with the dielectric constant 4.7 and thickness of $1.66 \mathrm{~mm}$. To fabricate a phase shifter on the PCB by means of chemical etching, a mask will first have to be made. In this case, the blueprint of the mask is made with AutoCAD software. The front side of the PCB was etched to achieve the desired pattern of the copper; the back side of the PCB was etched to remove all of the copper. Finally, the lumped elements were soldered onto the newly etched circuits. The varactors, resistors and inductors were soldered onto the circuit using a silver epoxy. Silver epoxy was used for two main reasons; it can hold the elements to the PCB board and it has a high conductivity. The phase shifter characteristics are controlled mainly by the properties of the varactor according to (5). In the phase shifter 


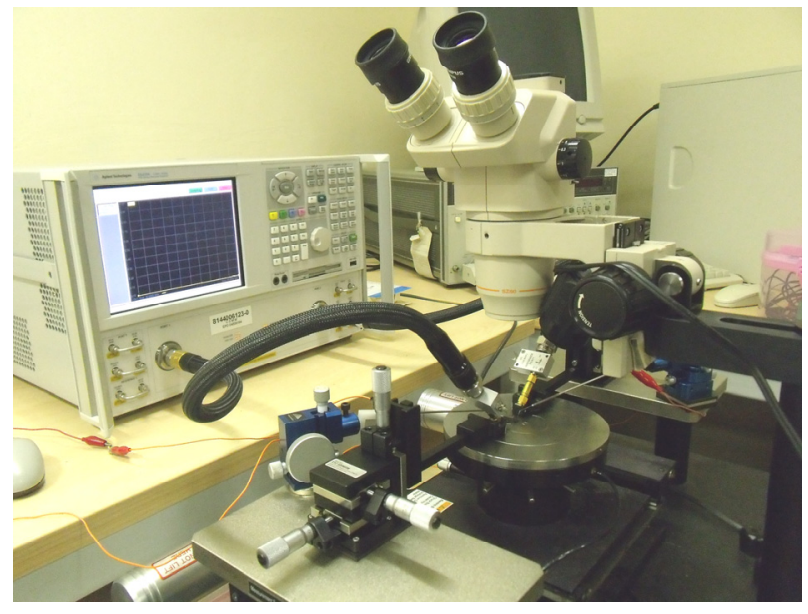

Fig. 6. The photograph of microwave measurement setup. Two ground-signal-ground (GSG) coplanar waveguide microprobes (GGB Industries, Model 40A) mounted on XYZ stages were used for this purpose. Pasternack PE1611 bias tee was used to supply DC bias voltage on picoprobe.

circuit, GaAs constant gamma flip-chip varactor diodes (M/A-COM's MA46H120 model) were used as series nonlinear capacitance. The dc bias wires were connected using $3 \mathrm{k} \Omega$ resistors between the varactor diodes. The fabricated varactor loaded transmission line metamaterial phase shifter is shown in Fig. 5. The dc bias voltage can be easily supplied between the copper pad, which is connected with shunt resistors between two varactors, and the signal probe.

The scattering matrix of the fabricated transmission line metamaterial phase shifter was measured by PNA Series Network Analyzer with a SOLT calibration, as shown in Fig. 6. The dc electrical fields from $0 \mathrm{~V}$ to $6 \mathrm{~V}$ were applied via signal probe and the copper pad. All measurements were taken from $7.2 \mathrm{GHz}$ to $8.7 \mathrm{GHz}$. The insertion loss and return loss of the phase shifter at $0 \mathrm{~V}$ and $6 \mathrm{~V}$ biases are shown in Fig. 7. A maximum insertion loss of $3.5 \mathrm{~dB}$, a minimum of $1.5 \mathrm{~dB}$, and a minimum return loss of $12 \mathrm{~dB}$ across all phase states over $7.2 \mathrm{GHz}$ to 8.7 GHz. The small variation in the magnitude of the insertion loss during tuning of the transmission line is a required property for antenna beam forming applications where a constant magnitude for all phases is recommended. Comparing Fig. 7 with Fig. 4, it can be observed that the fabricated transmission line metamaterial phase shifter retains its good impedance matching and transmission characteristics even though the reflection coefficient is significantly changed from simulated result to measured result. The measured return loss deviates by about $16 \%$ from the predicted simulated value. This error can be associated with the fabrication anomalies and silver epoxy with relatively low conductivity. The used simulation model provides a qualitative prediction of the performance, but an exact match in S11 and S21 should not be expected. Figure 8 shows measured differential phase shift with respect to the zero bias state as a function of frequency under different bias voltages. The circuit was capable of a $0^{\circ}-190^{\circ}$ continuous phase shift at $7.2 \mathrm{GHz}$ from $0 \mathrm{~V}$ to $6 \mathrm{~V}$. This

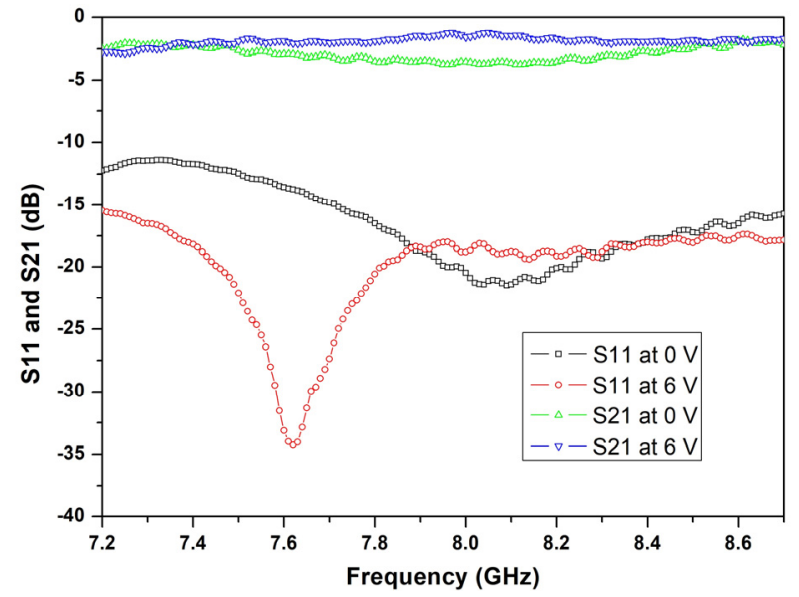

Fig. 7. Frequency dependent insertion loss and return loss of the transmission line metamaterial phase shifter at $0 \mathrm{~V}$ and $6 \mathrm{~V}$ bias voltages, respectively.

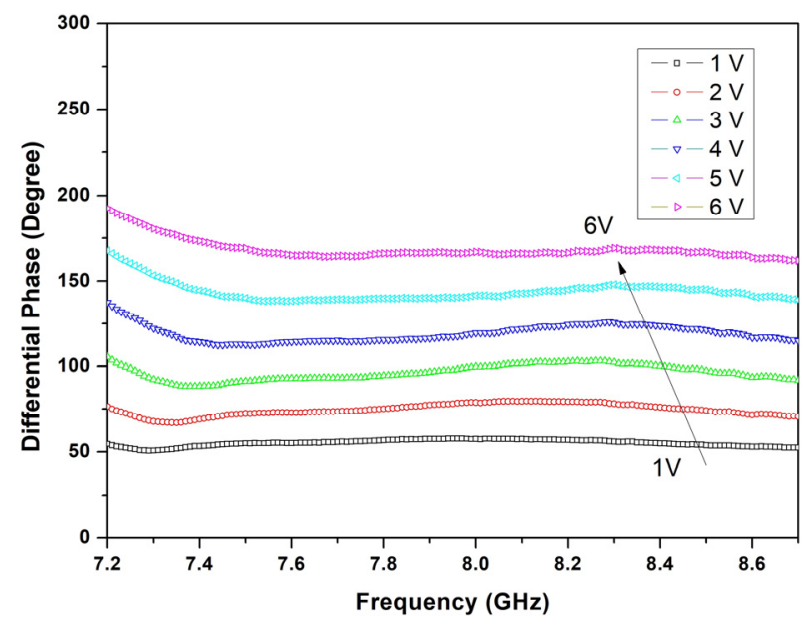

Fig. 8. Differential phase shift at different bias voltages as a function of frequency.

corresponds to a figure of merit of $F=\sim 57^{\circ} / \mathrm{dB}$, where $F$ is defined as the maximum differential phase shift divided by the insertion loss averaged across the full voltage range, at the operating frequency. As shown in Tab. 1, it is seen that the reflection loss of the left-hand transmission line phase shifter fabricated in our group is better than that reported in the reference [28]. Also, the fabricated device has larger differential phase shift than the reported one $\left(52^{\circ} \sim 58^{\circ}\right.$ at $17 \mathrm{GHz}$ ). The proposed LH phase shifter circuit achieved low loss variation, low return loss, negligible power consumption, and very linear phase variation with voltage. The figure of merit can be further improved by applying higher electric field. It demonstrates that this circuit structure has high potential for phase shift keying applications.

\section{Conclusions}

A broadband tunable microwave phase shifter using varactor loaded transmission line metamaterial has been proposed, which offers a great bandwidth advantage and has a relatively small insertion loss. Ansoft's HFSS was used to design and analyze a transmission line metamate- 
rial phase shifter structure, and the resulting S-parameters were used to characterize its performance. Based on the simulation result, a transmission line metamaterial phase shifter was fabricated on Copper-Clad Board. The designed four unit-cells phase shifter provides a $0-190^{\circ}$ continuous phase shift at $7.2 \mathrm{GHz}$ using varactors biased from $0 \mathrm{~V}$ to $6 \mathrm{~V}$ with relatively low insertion loss. Measured figure of merit is about $57^{\circ} / \mathrm{dB}$ using the highest insertion loss. The useable bandwidth of the proposed device for which the $|\mathrm{S} 11|$ of both states simultaneously remains below $-10 \mathrm{~dB}$ is $1.5 \mathrm{GHz}$, from $7.2 \mathrm{GHz}$ to $8.7 \mathrm{GHz}$. The measured results of the phase shifter show that in the whole pass band, the structure can maintain the phase shift very well and has relatively low insertion loss. Further work will be also required to improve the transmission characteristics of the proposed LH phase shifter. In the future, there will be more flexible metamaterial structure which can be easily integrated with other microwave components and devices. It is ideal for broadband application in microwave and RF circuit, such as wideband zeroth order resonator antenna and wideband series power divider.

\section{Acknowledgments}

This work was supported by the National Natural Science Foundation of China (Grant No. 61302046) and the Natural Science Foundation of Hubei Province of China (Grant No. 2019CFB435), and the Undergraduate research project of Hubei Normal University (Grant No. 2019077).

\section{References}

[1] VESELAGO, V. G. The electrodynamics of substances with simultaneously negative values of $\varepsilon$ and $\mu$. Soviet Physics-Uspekhi, 1968, vol. 10, no. 4, p. 509-514. DOI: 10.1070/PU1968v010n04ABEH003699

[2] SMITH, D. R., PADILLA, W. J., VIER, D. C., et al. Composite medium with simultaneously negative permeability and permittivity. Physical Review Letters, 2000, vol. 84, no. 18, p. 4184-4187. DOI: 10.1103/physrevlett.84.4184

[3] CHEN, H. S., RAN, L. X., HUANGFU, J. T., et al. Left-handed materials composed of only S-shaped resonators. Physical Review E, 2004, vol. 70, no. 5, p. 1-4. DOI: 10.1103/PhysRevE.70. 057605

[4] SANADA, A., CALOZ, C., ITOH, T. Characteristics of the composite right/left-handed transmission lines. IEEE Microwaves and Wireless Components Letters, 2004, vol. 14, no. 2, p. 68-70. DOI: $10.1109 / \mathrm{lmwc} .2003 .822563$

[5] MAO, S. G., CHEN, S. L., HUANG, C. W. Effective electromagnetic parameters of novel distributed left-handed microstrip lines. IEEE Transactions on Microwave Theory and Techniques, 2005, vol. 53, no. 4, p. 1515-1521. DOI: $10.1109 /$ tmtt.2005.845192

[6] LI, N., YU, H., YANG, C., et al. A high-sensitivity $135 \mathrm{GHz}$ millimeter-wave imager by compact split-ring-resonator in $65-\mathrm{nm}$
CMOS. Solid-State Electronics, 2015, vol. 113, p. 54-60. DOI: 10.1016/j.sse.2015.05.006

[7] MARTIN, F., FAlCONE, F., BONACHE, J., et al. Split ring resonator based left handed coplanar waveguide. Applied Physics Letters, 2003, vol. 83, p. 4652-4654. DOI: 10.1063/1.1631392

[8] FALCONE, F., LOPETEGI, T., BAENA, J. D., et al. Effective negative-epsilon stop-band microstrip lines based on complementary split ring resonators. IEEE Microwave and Wireless Components Letters, 2004, vol. 14, p. 280-282. DOI: 10.1109/lmwc.2004.828029

[9] GIL, I., GARCIA-GARCIA, J., BONACHE, J., et al. Varactorloaded split rings resonators for tuneable notch filters at microwave frequencies, Electronics Letters, 2004, vol. 40, p. 1347-1348. DOI: 10.1049 /el:20046389

[10] VELEZ, A., BONACHE, J., MARTIN, F. Varactor-Loaded Complementary Split Ring Resonators (VLCSRR) and their application to tunable metamaterial transmission lines. IEEE Microwave and Wireless Components Letters, 2008, vol. 18, no. 1, p. 28-30. DOI: $10.1109 / \mathrm{lmwc} .2007 .911983$

[11] IYER, A. K., ELEFTHERIADES, G. V. Negative refractive index metamaterials supporting 2-D waves. In IEEE MTT-S International Microwave Symposium Digest. Seattle (WA, USA), 2002, p. 1067-1070. DOI: 10.1109/mwsym.2002.1011823

[12] CALOZ, C., ITOH, T. Application of the transmission line theory of left-handed ( $\mathrm{LH})$ materials to the realization of a microstrip $\mathrm{LH}$ transmission line. In IEEE Antennas and Propagation Society International Symposium. San Antonio (TX, USA), 2002, vol. 2, p. 412-415. DOI: $10.1109 /$ APS.2002.1016111

[13] OLINER, A. A. A periodic-structure negative-refractive-index medium without resonant elements. In IEEE Antennas and Propagation Society International Symposium. San Antonio (TX, USA), 2002, p. 41.

[14] GIERE, A., DAMM, C., SCHEELE, P., JAKOBY, R. LH phase shifter using ferroelectric varactors. In Proceeding of the IEEE Radio and Wireless Symposium. San Diego (CA, USA), 2006, p. 403-406. DOI: 10.1109 /RWS.2006.1615180

[15] CALOZ, C., SANADA, A., ITOH, T. A novel composite right/left-handed coupled-line directional coupler with arbitrary coupling level and broad bandwidth. IEEE Transactions on Microwave Theory and Techniques, 2004, vol. 52, no. 3, p. 980-992. DOI: $10.1109 /$ tmtt.2004.823579

[16] KHOLODNYAK, D., ZAMESHAEVA, E., TURGALIEV, V., et al. Tunable dual-frequency immittance inverters on dual-composite right/left handed transmission lines (D-CRLH TL) with variable capacitors. IEICE Transactions on Electronics, 2016, vol. E99-C, no. 10 , p. $1113-1121$. DOI: $10.1587 /$ transele.e99.c.1113

[17] JACKSON, D., CALOZ, C., ITOH, T. Leaky-wave antennas. Proceedings IEEE, 2012, vol. 100, no. 7, p. 2194-2206. DOI: 10.1109/JPROC. 2012.2187410

[18] CHOI, J., SEO, C. Broadband VCO using electronically controlled metamaterial transmission line based on varactor-loaded split-ring resonator. Microwave and Optical Technology Letters, 2008, vol. 50, no. 4, p. 1078-1082. DOI: 10.1002/mop.23305

[19] OURIR, A., ABDEDDAIM, R., DE ROSNY, J. Tunable trapped mode in symmetric resonator designed for metamaterials. Progress In Electromagnetics Research, 2010, vol. 101, p. 115-123. DOI: 10.2528/pier09120709

[20] POWELl, D. A., SHADRIVOV, I. V., KIVSHAR, Y. S. Asymmetric parametric amplification in nonlinear left-handed transmission lines. Applied Physics Letters, 2009, vol. 94, p. 1-3. DOI: $10.1063 / 1.3089842$

[21] WANG, Z. B., FENG, Y. J., ZHU, B., et al. Dark Schrodinger solitons and harmonic generation in left-handed nonlinear transmis- 
sion line. Journal of Applied Physics, 2010, vol. 107, p. 1-5. DOI: $10.1063 / 1.3418556$

[22] CHOI, S., SU, W., TENTZERIS, M. M., et al. A novel fluidreconfigurable advanced and delayed phase line using inkjetprinted microfluidic composite right/left-handed transmission line. IEEE Microwaves and Wireless Components Letters, 2015, vol. 25 , no. 2 , p. $142-144$. DOI: $10.1109 / 1 m w c .2014 .2382685$

[23] MICHISHITA, N., KITAHARA, H., YAMADA, Y., et al. Tunable phase shifter using composite right/left-handed transmission line with mechanically variable MIM capacitors. IEEE Antennas and Wireless Propagation Letters, 2011, vol. 10, p. 1579-1581. DOI: 10.1109/lawp.2011.2181147

[24] ABDAlla, M. A. Y., PHANG, K, ElEFTHERIADES, G. V. Printed and integrated CMOS positive/negative refractive-index phase shifters using tunable active inductors. IEEE Transactions on Microwave Theory and Techniques, 2007, vol. 55, no. 8, p. 1611-1623. DOI: $10.1109 / \mathrm{tmtt} .2007 .901076$

[25] DURAN-SINDREU, M., DAMM, C., SAZEGAR, M., et al. Electrically tunable composite right/left handed transmission-line based on open resonators and barium-stronium-titanate thick films. In IEEE MTT-S International Microwave Symposium. Baltimore (MD, USA), 2011, p. 1-4. DOI: 10.1109/mwsym.2011.5973393

[26] KIM, H., KOZYREV, A. B., KARBASSI, A., et al. Compact lefthanded transmission line as a linear phase-voltage modulator and efficient harmonic generator. IEEE Transactions on Microwave Theory and Techniques, 2007, vol. 55, no. 3, p. 571-578. DOI: $10.1109 /$ tmtt.2007.891692

[27] DAMM, C., SCHUSSLER, M., OERTEL, M., et al. Compact tunable periodically LC loaded microstrip line for phase shifting applications. In IEEE MTT-S International Microwave Symposium. Long Beach (CA, USA), 2005, p. 2003-2006. DOI: 10.1109/mwsym.2005.1517137

[28] KUYLENSTIERNA, D., VOROBIEV, A., LINNER, P., et al. Composite right/left handed transmission line phase shifter using ferroelectric varactors. IEEE Microwaves and Wireless Components Letters, 2006, vol. 16, no. 4, p. 167-169. DOI: $10.1109 /$ lmwc.2006.872145

[29] PERRUISSEAU-CARRIER, J., TOPALLI, K., AKIN, T. Low-loss Ku-band artificial transmission line with MEMS tuning capability. IEEE Microwave and Wireless Components Letters, 2009, vol. 19, no. 6, p. 377-379. DOI: 10.1109/lmwc.2009.2020022

[30] ABBASI, M. A. B., ANTONIADES, M. A., NIKOlaOU, S. A compact reconfigurable NRI-TL metamaterial phase shifter for antenna applications. IEEE Transactions on Antennas and Propagation, 2018, vol. 66, no. 2, p. 1025-1030. DOI: 10.1109/TAP.2017.2777520

[31] ELLINGER, F., JACKEL, H., BACHTOLD, W. Varactor-loaded transmission-line phase shifter at C-band using lumped elements. IEEE Transactions on Microwave Theory and Techniques, 2003, vol. 51, no. 4, p. 1135-1140. DOI: 10.1109/TMTT. 2003.809670
[32] CALOZ, C., ITOH, T. Transmission line approach of left-handed (LH) materials and microstrip implementation of an artificial $\mathrm{LH}$ transmission line. IEEE Transactions on Antennas and Propagation, 2004, vol. 52, no. 5, p. 1159-1166. DOI: 10.1109/TAP.2004.827249

[33] SALEH, B. A., TEICH, M. C. Fundamentals of Photonics. New York: Wiley, 1991. DOI:10.1002/0471213748

\section{About the Authors...}

Su SHENG received the M.S. degree in 2006 from Wuhan University, Wuhan, China and the Ph.D. degree in 2012 from the National University of Singapore, Singapore. He is currently working as an associate professor of the College of Physics and Electronic Science, Hubei Normal University. His research interests include ferroelectric materials and its microwave applications, microwave measurement and electromagnetic metamaterials.

Hanxiao CHEN received the B.S. degree from the College of Technology and Engineering, Lanzhou University of Technology, Lanzhou China, in 2017. She is currently working toward M.S. degree at the College of Physics and Electronic Science, Hubei Normal University. Her main interests include microwave devices based on ferroelectric materials and electromagnetic metamaterials.

Jian WEN received Ph.D. degree in New Energy Nanomaterial Field at the School of Physical Science and Technology, Wuhan University, Wuhan, China, in 2017. He is currently working as an instructor in the College of Physics and Electronic Science, Hubei Normal University since 2017. His research activity has been focused on the nanomaterial and device structure design in energy storage device including supercapacitor, nickel-zinc battery and HER catalysis etc.

Hongri LIU received the B.S. and M.S. degrees from Hubei Normal University and Hubei University in 1995 and 1998, respectively. He received Ph.D. degree from the School of Physics, Huazhong University of Science and Technology in 2006. He joined Hubei Normal University in 1998 as an assistant teacher. He is currently a professor of Hubei Normal University. His research interests include multiferroics and organic perovskite photovoltaic materials and devices. He has authored more than 30 articles and conference papers. 